\title{
Production of Putrescine and Cadaverine by Paucilactobacillus wasatchensis
}

\author{
Hélène Berthoud*, Daniel Wechsler ${ }^{\dagger}$ and Stefan Irmler \\ Agroscope, Bern, Switzerland
}

OPEN ACCESS

Edited by:

Claudia Ruiz-Capillas, Institute of Science and Technology of Food and Nutrition (CSIC), Spain

Reviewed by:

Chiara Montanari, University of Bologna, Italy

Zhen Feng,

Northeast Agricultural University,

China

*Correspondence:

Hélène Berthoud

helene.berthoud@agroscope.admin.ch

${ }^{\dagger}$ Deceased

Specialty section: This article was submitted to

Food Microbiology, a section of the journal

Frontiers in Microbiology

Received: 23 December 2021 Accepted: 14 February 2022

Published: 03 March 2022

Citation:

Berthoud H, Wechsler D and Irmler S (2022) Production of

Putrescine and Cadaverine by Paucilactobacillus wasatchensis. Front. Microbiol. 13:842403. doi: 10.3389/fmicb.2022.842403
Lactic acid bacteria ( $L A B)$ play a key role in many food fermentations. However, some $\angle A B$ species can also cause food spoilage, e.g., through the formation of biogenic amines. Paucilactobacillus wasatchensis is a LAB that causes late gas production in Cheddar cheese, the molecular causes of which are not fully understood. This study reports on the ability of $P$. wasatchensis WDCO4 to produce cadaverine and putrescine in broth supplemented with lysine and ornithine, as well as in a model cheese. The raclette-type semi-hard cheese produced with $P$. wasatchensis as an adjunct culture contained $1,085 \mathrm{mg} \mathrm{kg}^{-1}$ of cadaverine and $304 \mathrm{mg} \mathrm{kg}^{-1}$ of putrescine after 120 days of ripening. We identified two ornithine decarboxylase genes (odc) and a putrescine-ornithine antiporter gene (potE) in the genome sequence of $P$. wasatchensis. We could show that the two odc genes, which are located on two contigs, are contiguous and form the genetic cluster odc2-odc1-potE. Alignment searches showed that similar gene clusters exist in the genomes of Levilactobacillus paucivorans DSMZ22467, Lentilactobacillus kribbianus $\mathrm{YH}-\mathrm{lac}$, Levilactobacillus hunanensis 151-2B, and Levilactobacillus lindianensis 220-4. More amino acid sequence comparisons showed that Odc1 and Odc2 shared 72 and $69 \%$ identity with a lysine and ornithine decarboxylase from Ligilactobacillus saerimneri 30a, respectively. To clarify the catalytic activities of both enzymes, the odc-coding genes were cloned and heterologously expressed as His-tagged fusion protein. The purified Odc1 protein decarboxylated lysine into cadaverine, while the recombinant Odc2 protein preferentially produced putrescine from ornithine but also exhibited low lysine decarboxylating activity. Both enzymes were active at $\mathrm{pH}$ of 5.5 , a value often found in cheese. To our knowledge, this is only the second lysine decarboxylase in LAB whose function has been verified. The tandem arrangement of the genes in a single cluster suggests a gene duplication, evolving the ability to metabolize more amino. Divergent substrate preferences highlight the necessity of verifying the functions of genes, in addition to automatic annotation based on sequence similarity. Acquiring new biochemical data allows better predictive models and, in this case, more accurate biogenic amine production potential for $L A B$ strains and microbiomes.

Keywords: Paucilactobacillus wasatchensis, Lactobacillus, putrescine, cadaverine, ornithine decarboxylase, lysine decarboxylase, cheese, biogenic amines 


\section{INTRODUCTION}

Fermented foods are defined as foods or beverages produced through controlled microbial growth and the conversion of food components through enzymatic action (Dimidi et al., 2019). Their effect on health is considered positive, despite very limited clinical evidence for such a claim (Gille et al., 2018; Dimidi et al., 2019). Fermentation is also used to enhance organoleptic properties. Cheeses made with raw milk have been shown to contain greater microbial diversity, ripen faster, and develop a richer and more intense flavor than pasteurized milk cheeses (Montel et al., 2014; Egger et al., 2021). However, fermentation may also produce undesired metabolites, such as biogenic amines. The most important BAs in cheese are histamine and tyramine (produced via the enzymatic decarboxylation of histidine and tyrosine, respectively), putrescine (synthesized via ornithine decarboxylation or agmatine deamination), and, more rarely, cadaverine (originated by lysine decarboxylation; Linares et al., 2011). In hard and semi-hard cheeses, the accumulation of histamine is generally associated with Lentilactobacillus parabuchneri (O'Sullivan et al., 2015; Berthoud et al., 2017; Tofalo et al., 2019). Enterococci are considered tyramine formers, but other lactobacilli, such as Latilactobacillus curvatus and Levilactobacillus brevis, have also been shown to produce high levels of tyramine (Cid et al., 2008; Coton and Coton, 2009; Schirone et al., 2013). Enterobacteria are known to produce cadaverine and putrescine (Coton et al., 2012; Schirone et al., 2012); however, their population density typically decreases considerably in hard and semi-hard cheeses within the first 90 days of ripening (Bachmann and Spahr, 1995; Maher et al., 2001; Rios et al., 2020). In the core of an uncooked pressed type model cheese inoculated with Gram-negative cadaverine producers, only low amounts of cadaverine were measured ( $<100 \mathrm{mg} \mathrm{kg}^{-1}$ cheese dry matter; Delbès-Paus et al., 2012). Regarding the extreme levels of cadaverine $\left(>1,000 \mathrm{mg} \mathrm{kg}^{-1}\right)$ reported by Schirone et al. (2013) and Benkerroum (2016) in Pecorino di Fossa, a semi-hard cheese, other microorganisms may be responsible (Linares et al., 2011).

Lactic acid bacteria (LAB) include many species, and some of them are able to grow in cheese during ripening. Moreover, some strains have been shown to produce biogenic amines and contain amino acid-decarboxylating genes (Cid et al., 2008; Romano et al., 2014; Zhang and Ni, 2014; Del Rio et al., 2018). In particular, Ligilactobacillus saerimneri 30a was shown to be capable of decarboxylating histidine, ornithine, and lysine into histamine, putrescine, and cadaverine, respectively (Pessione et al., 2005). Romano et al. sequenced the genome and identified a three-component lysine/ornithine decarboxylation system (Romano et al., 2013a,b) composed of an amino acid transporter (PotE; RefSeq WP_009553966) that had affinity for both the lysine/cadaverine and ornithine/putrescine pairs, an ornithine decarboxylase (Odc; RefSeq WP_009553942) with an additional lower affinity for lysine, and a new specific lysine decarboxylase (Ldc; RefSeq WP_009553967). In fact, Odc and Ldc shared $59.4 \%$ amino acid identity and short conserved sequences. Previously, the authors also described putative arginine/ornithine/ lysine decarboxylases (Aoldc) that were widespread in LAB and were not associated with an ornithine/putrescine exchanger. The Aoldc proteins from Lacticaseibacillus paracasei (RefSeq WP_011674560) and Lactobacillus gasseri (RefSeq WP_011679015) had a neutral pH optimum but very low activities with ornithine and lysine as substrates. In L. saerimneri, Aoldc (RefSeq WP_040533848) shared 49.4 and $47.4 \%$ amino acid identity with Ldc and Odc, respectively, as well as having short conserved domains. The similarities between the different decarboxylase proteins make automatic annotations and function predictions tricky. However, in performing a BLAST search using the amino sequence of this lysine decarboxylase as query, one interesting hit was the sequence RefSeq WP_044011228 from Paucilactobacillus wasatchensis, which was annotated as ornithine decarboxylase and shared $72 \%$ identity with the $\mathrm{Ldc}$ of L. saerimneri.

Additionally, $P$. wasatchensis sp. nov. was isolated from aged Cheddar (Oberg et al., 2016). This bacterium is a slowgrowing obligatory heterofermentative lactobacillus that grows at storage temperatures between 6 and $12^{\circ} \mathrm{C}$ and up to $30^{\circ} \mathrm{C}$. Its growth and survival characteristics allow it to grow in cheese (McMahon et al., 2020), and it was shown to be associated with late gas production in Cheddar cheese during storage (Ortakci et al., 2015b).

The aims of this study were to (i) investigate the capacity of $P$. wasatchensis WDC04 (DSM 29958) to produce cadaverine and putrescine by decarboxylating lysine and ornithine in culture medium, as well as in a trial cheese; (ii) identify the putative amino acid decarboxylase genes; and (iii) evaluate the decarboxylation activity of recombinant proteins, as well as their substrate preference. A raclette-type semi-hard model cheese with adjunct culture of $P$. wasatchensis was produced. After 120 ripening days, the composition of the free amino acids and the biogenic amine content were determined. The quantification of $P$. wasatchensis in cheese was performed by a TaqMan real-time PCR assay designed based on the DNA recombinase A recA gene.

\section{MATERIALS AND METHODS}

\section{Growth Conditions and Formation of Cadaverine and Putrescine}

P. wasatchensis, formerly Lactobacillus wasatchensis, DSM 29958 obtained from the German Collection of Microorganisms and Cell Cultures GmbH (DSMZ, Braunschweig, Germany) was cultivated in modified MRS broth (mMRS) at $25^{\circ} \mathrm{C}$. This medium was prepared according to the cultivation conditions recommended by the DSMZ. This means that MRS (Man et al., 1960) was supplemented with $0.05 \%$ L-cysteine and $1.5 \%$ D-ribose, and the $\mathrm{pH}$ was adjusted to 5.2. To evaluate the capability to produce cadaverine and putrescine, the DSM 29958 strain was incubated in mMRS supplemented with $1 \% \mathrm{~L}$-lysine, $1 \% \mathrm{~L}$-ornithine, or $1 \% \mathrm{~L}$-arginine at $25^{\circ} \mathrm{C}$ for 4 days. Biogenic amines were derivatized with dansyl chloride, extracted, and separated using high-performance thin-layer chromatography (HPTLC) as described by $\mathrm{Li}$ et al. (2016). In contrast to $\mathrm{Li}$ et al., the dansylated amines were extracted with ethyl acetate 
rather than diethyl ether. The solvent system consisted of chloroform-triethylamine (4:1, v:v).

\section{Protein Sequence Analysis}

Database searches were performed using the BLAST programs developed by Altschul et al. (1997). Sequences were aligned with CLUSTAL Omega (1.2.4; Sievers et al., 2011). The alignment was employed to generate and visualize an unrooted tree using SEAVIEW 5.0.4 (Gouy et al., 2010), applying the neighborjoining (NJ) method, and the Kimura two-parameter model (K2P).

\section{Production of the Model Cheese With an Adjunct Culture of $P$. wasatchensis}

A raclette-type semi-hard model cheeses to which $P$. wasatchensis DSM 29958 was added as an adjunct and a control cheese without the strain were manufactured in the experimental cheese dairy at Agroscope (Bern, Switzerland) as described by Dreier et al. (2020). Briefly, the cheeses were produced from $50 \mathrm{~L}$ of pasteurized milk using a combination of the mesophilic starter RSW 901 (Lactococcus lactis subsp. lactis, L. lactis subsp. cremoris, and $L$. lactis subsp. diacetylactis) and the mixed mesophilic/thermophilic starter MK 401 (L. lactis subsp. lactis, Lactobacillus delbrueckii subsp. lactis, and Streptococcus thermophilus; Liebefeld Kulturen AG, Switzerland). The estimated concentration of the adjunct culture in the milk vat was $10^{4}-10^{5} \mathrm{CFU} \mathrm{ml}{ }^{-1}$. The cheeses $(30 \mathrm{~cm}$ in diameter, about $6 \mathrm{~kg})$ were immersed in a $20 \%(\mathrm{w} / \mathrm{w})$ saline solution $\left(11-13^{\circ} \mathrm{C}, 14 \mathrm{~h}\right)$ and smear-ripened in a maturing cellar $\left(10-11^{\circ} \mathrm{C}, 90-96 \%\right.$ relative humidity) for 120 days.

\section{Chemical Analysis of Cheeses}

Free amino acid concentrations were determined using highperformance liquid chromatography (HPLC), as previously described (Wenzel et al., 2018). Biogenic amines were derivatized with dansyl chloride prior to ultra-performance liquid chromatography (UPLC) separation, as previously described (Ascone et al., 2017).

\section{DNA Extraction and Species-Specific qPCR}

The DNA was extracted from a pure culture of P. wasatchensis, as well as from the cheeses, as described by Berthoud et al. (2017). For the species-specific quantitative real-time PCR (qPCR), primers and probe were designed based on the gene recA, which codes for the DNA recombinase A protein (RefSeq NZ_AWTT01000028 REGION: 23402.24457). The assays were performed in a reaction volume of $12 \mu$ c containing $6 \mu$ of Takyon $^{\mathrm{TM}}$ No ROX Probe 2X MasterMix UNG (Eurogentec, Seraing, Belgium), $300 \mathrm{nM}$ of forward (5'-CGAATTG GTCAAGGTCGAGAA- $3^{\prime}$ ) and reverse primer (5'-GACCTTTTG CATTAACTCATCCATT-3'), $100 \mathrm{nM}$ of hydrolysis probe (5'-FAMTGCCAAACAATATTTCGCTGATCATCCAG-BHQ1-3'), and $2 \mu \mathrm{l}$ of DNA. The qPCR conditions were $50^{\circ} \mathrm{C}$ for $2 \mathrm{~min}$ and $95^{\circ} \mathrm{C}$ for $3 \mathrm{~min}$, followed by 40 cycles of $95^{\circ} \mathrm{C}$ for $3 \mathrm{~s}$ and $60^{\circ} \mathrm{C}$ for $20 \mathrm{~s}$. All qPCR assays were run on a Corbett Rotor-Gene
3000 (Qiagen, Hilden, Germany), and a serial dilution of the genomic DNA of strain DSM 29958 was included in each run. The DNA concentration was determined using a NanoDrop ${ }^{\circledR}$ ND-1000 Spectrophotometer (NanoDrop Technologies, Thermo Fisher Scientific, Waltham, MA, United States). The number of copies, corresponding to DNA concentration, was estimated using $660 \mathrm{pmol} \mathrm{gg}^{-1}$ as the average molecular weight for a nucleotide pair and $1.90425 \mathrm{Mb}$ as the genome size of $P$. wasatchensis. The analysis was performed using Rotor-Gene Q Series Software v2.3.1 (Qiagen), using a threshold of 0.05 for the quantification cycle $(\mathrm{Cq})$ value determination.

\section{Cloning of Putative Decarboxylases Genes odc1 and odc2}

The genes coding for the putative ornithine decarboxylases $o d c 1$ and $o d c 2$ from $P$. wasatchensis were amplified with primers containing a restriction site for BamHI and SalI (restriction sites are underlined). The primers were designed using the sequences of the odc1 gene (odc1_F 5'-ATATGGA TCCAATGAAATTATTAGGTATCGCGT-3'; odc1_R 5'-TCGAG TCGACCGTTTTTCTTTATTACAGATTCATCA-3') and odc2 gene (odc2_F 5'-ATATGGATCCAATGAATAGTATGAAAATT GCTACAA-3'; odc2_R 5'-TCGAGTCGACCTTTTTGCTCTT CTAGCACGTA-3'). Amplification reactions were performed in a final volume of $50 \mu \mathrm{l}$, containing $25 \mu \mathrm{l}$ of $2 \times$ Platinum $^{\mathrm{TM}}$ SuperFi ${ }^{\mathrm{TM}}$ PCR Master Mix (Invitrogen, Thermo Fisher Scientific), $2.5 \mu \mathrm{l}$ of each primer at $0.5 \mathrm{mM}$, and $25 \mathrm{ng}$ of genomic DNA. Amplifications were carried out in a thermocycler (Applied Biosystems, Thermo Fisher Scientific) with the following program: initial denaturation at $98^{\circ} \mathrm{C}$ for $30 \mathrm{~s}$, followed by 5 cycles at $98^{\circ} \mathrm{C}$ for $10 \mathrm{~s}, 52^{\circ} \mathrm{C}$ for $10 \mathrm{~s}$, and $72^{\circ} \mathrm{C}$ for $2 \mathrm{~min}$; then, there were 30 cycles under the same conditions but with an annealing temperature of $65^{\circ} \mathrm{C}$, as well as a final prolongation of $5 \mathrm{~min}$ at $72^{\circ} \mathrm{C}$. The DNA fragments obtained were digested with enzymes and then ligated with T4 DNA ligase (Invitrogen) into the pEG-His1 (MoBiTec Molecular Biotechnology, Goettingen, Germany) vector previously digested with the same enzymes. One-Shot ${ }^{\mathrm{TM}}$ Mach1 chemically competent E. coli (Invitrogen) was transformed with the ligation product. The positive clones obtained were named Odc1_Lw35 and Odc2_C19, respectively.

\section{Production and Purification of Recombinant Proteins}

The expression plasmids were transformed into One-Shot ${ }^{\mathrm{TM}}$ BL21(DE3) chemically competent E. coli (Invitrogen) according to the manufacturer's instructions. The transformation reaction was plated into $\mathrm{LB}$ agar plates containing $0.1 \mathrm{mg} / \mathrm{ml}$ ampicillin. After incubation overnight at $37^{\circ} \mathrm{C}$, a colony was transferred into $100 \mathrm{ml}$ of MagicMedia ${ }^{\mathrm{TM}}$ E. coli expression medium (Thermo Fisher Scientific). The medium was then incubated at $30^{\circ} \mathrm{C}$ for $20 \mathrm{~h}$ on a shaker $(220 \mathrm{rpm})$.

The bacterial cells were harvested via centrifugation $(3,000 \mathrm{~g}$, RT, $10 \mathrm{~min}$ ), washed once with $20 \mathrm{mM}$ of sodium phosphate ( $\mathrm{pH}$ 7.4), and frozen at $-20^{\circ} \mathrm{C}$. The recombinant, his-tagged protein was purified using a Protino Ni-TED 1000 Kit (Machery-Nagel, 
Oensingen, Switzerland) according to the manufacturer's protocol. The buffer of the eluate containing the recombinant protein was exchanged with $20 \mathrm{mM}$ of sodium phosphate $(\mathrm{pH}$ 7.4) using illustra $^{\mathrm{TM}} \mathrm{NAP}^{\mathrm{TM}}$ columns (VWR International GmbH, Dietikon, Switzerland) according to the manufacturer's protocol. The protein concentration was determined using the Qubit Protein Assay Kit (Thermo Fisher Scientific). The purity of the purified proteins was evaluated using denaturing polyacrylamide gel electrophoresis, followed by Coomassie Blue staining.

\section{Decarboxylase Activity Assay}

The above mentioned methods to determine biogenic amines qualitatively by HPTLC or quantitatively by HPLC cannot be used to detect amino acids. In order to observe both the amino acid substrates (lysine and ornithine) and amine (cadaverine and putrescine), a third method was used here. O-phthalaldehyde was used to derivatize amino acid and amine which were then separated and quantified by HPLC as previously described (Wenzel et al., 2018). This allowed to control that the substrate was not completely used up during the enzymatic reaction. The decarboxylation assay was performed in a volume of $200 \mu \mathrm{l}$. The assay consisted of $50 \mathrm{mM}$ phosphate buffer $(\mathrm{pH}$ $5.5), 50 \mu \mathrm{M}$ of pyridoxal $5^{\prime}$-phosphate, and $10 \mathrm{mM}$ of amino acid. L-Lysine, L-ornithine, and L-arginine were used as amino acids. The reaction was performed with $2 \mu \mathrm{g}$ of purified protein at $37^{\circ} \mathrm{C}$ for $30 \mathrm{~min}$. The exception was the decarboxylation of ornithine by Odc2_C19, for which only $0.2 \mu \mathrm{g}$ of enzyme was used and the reaction time was $10 \mathrm{~min}$.

For the determination of the kinetic parameters, the substrate concentration varied between 1 and $50 \mathrm{mM}$. Phosphate buffers with different $\mathrm{pH}$ s were used to determine the dependence on $\mathrm{pH}$.

The enzymatic reaction was stopped by adding $200 \mu \mathrm{l}$ of methanol. The sample was then filtered through a $0.45 \mu \mathrm{m}$ filter (nylon). The amines present in the filtrate were derivatized with O-phthalaldehyde and separated via HPLC, as described previously (Wenzel et al., 2018). Calibration standards were prepared with putrescine and cadaverine in 50\% methanol. The peak areas of putrescine and cadaverine were determined with Chromeleon software (v7.2.10, Thermo Fisher Scientific). The experimental data were fitted to the Michaelis-Menten equation using SciPy (Virtanen et al., 2020) to determine the $\mathrm{Km}$ and kcat.

\section{RESULTS}

\section{Formation of Biogenic Amines by Strain DSM 29958}

Cadaverine and putrescine were detected in all culture supernatants (Figure 1). Neither compound was present in the uninoculated medium. Upon the addition of lysine and ornithine, increased formations of cadaverine and putrescine, respectively, were observed. Medium without the addition of lysine allowed the production of cadaverine, probably due to low levels of lysine in the MRS broth. When the medium was supplemented with arginine, neither agmatine nor putrescine formation was observed.

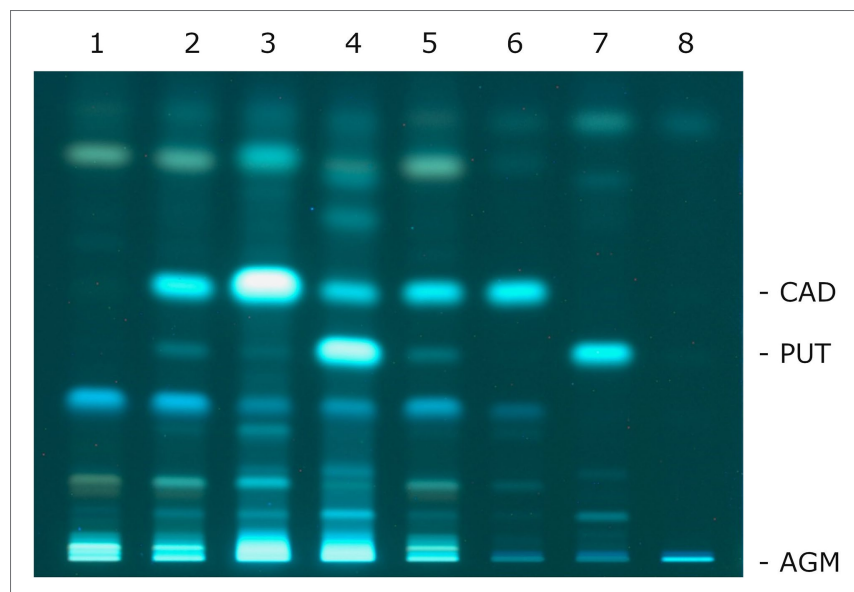

FIGURE 1 | Thin-layer chromatography of the biogenic amines presents in the culture supernatants of $P$. wasatchensis. 1: uninoculated medium; 2 : medium; 3: medium + lysine; 4: medium + ornithine; 5 : medium + arginine; 6 : cadaverine; 7: putrescine; and 8: agmatine.

\section{Determination of Biogenic Amines, Free Amino Acids, and $P$. wasatchensis in Model Cheeses}

The concentrations of biogenic amines (cadaverine, putrescine, histamine, and tyramine) and their amino acid precursors (lysine, ornithine, histidine, and tyrosine) were measured in a model cheese inoculated with $P$. wasatchensis, as well as in a control cheese, after 120 ripening days. Cadaverine and putrescine were detected at 1,085 and $304 \mathrm{mg} \mathrm{kg}^{-1}$ in cheese with $P$. wasatchensis, while the amounts of the respective amino acid precursors were reduced in comparison with the control cheese. No histamine and negligible concentrations of tyramine were detected. Amounts of histidine and tyrosine, as well as total free amino acids (FAAs), were similar in both cheeses (Table 1). P. wasatchensis was measured via qPCR in the inoculated cheese at an estimated concentration of $1.16 \times 10^{7}$ genome equivalents (GE) per gram, while no amplification signal was detected in the control cheese (Table 1). These results showed the concomitant growth of $P$. wasatchensis, the production of cadaverine and putrescine, as well as a reduction of the levels of lysine and ornithine as compared to the control cheese.

\section{Identifying and Cloning Two Putative Ornithine Decarboxylase Genes}

The genome sequence of $P$. wasatchensis strain WDC04 is deposited in the NCBI's Reference Sequence database (RefSeq NZ_AWTT00000000.1). This data entry contains 1773 proteins. It contains one complete ornithine decarboxylase and two partial ornithine decarboxylases. The complete one (WP_044011228) has a length of 726 amino acids, is located on contig000042, and is referred to as Odc1 in this report. Downstream of Odc1 is a putative putrescine-ornithine antiporter (PotE; WP_044011229). The two partial ornithine decarboxylases are found on contig000080 (WP_044011535) and contig000042 
TABLE 1 | Concentrations of biogenic amines $\left(\mathrm{mg} \mathrm{kg}^{-1}\right)$, free amino acids (FAAs, mmol $\mathrm{kg}^{-1}$ ), and $P$. wasatchensis (genome equivalent per g; GE $\mathrm{g}^{-1}$ ) in model cheeses ripened for 120 days.

Biogenic amines $\left(\mathrm{mg} \mathrm{kg}^{-1}\right)$ Free amino acid $\left(\mathrm{mmol} \mathrm{kg}^{-1}\right)$

P. wasatchensis (GE g-1)

\begin{tabular}{|c|c|c|c|c|c|c|c|c|c|c|}
\hline & & & \\
\hline & CAD & PUT & HIST & TYRA & Lys & Orn & Hist & Tyr & Total FAAs & \\
\hline $\begin{array}{l}\text { Cheese with } \\
\text { P. wasatchensis }\end{array}$ & 1,085 & 304 & ND & $<\mathrm{LOQ}$ & 1.05 & 0.26 & 2.82 & 3.16 & 193 & $7 \log$ \\
\hline Control cheese & $<\mathrm{LOQ}$ & ND & ND & $<\mathrm{LOQ}$ & 12.74 & 3.87 & 3.8 & 3.62 & 208 & ND \\
\hline
\end{tabular}

ND, not detected; LOQ, limit of quantitation; CAD, cadaverine; PUT, putrescine; HIST, histamine; TYRA, tyramine; Lys, lysine; Orn, ornithine; Hist, histidine; and Tyr, tyrosine.

odc2 (OL347710)
FIGURE 2 I Schematic illustration of the genetic region involved in lysine and ornithine decarboxylation in $P$. wasatchensis. The green and red arrows represent the
RefSeq accession numbers of the genomic and protein data, respectively. The blue arrows represent the odc1 and odc2 genes that were cloned in this study. The
gray arrow represents the ornithine-putrescine antiporter gene potE.

(WP_044011227), respectively. WP_044011227 has a length of 60 amino acids. We suspected that this is the missing part of the protein WP_044011535, which has a length of 660 amino acids, and that an assembly error likely caused the coding sequence to be spread over two contigs. To test this hypothesis, an attempt was made to amplify the junction sequence of both contigs via PCR.

Therefore, the primers Lwasa_odc2_F 5'-CTTCCAGAA CCAGCAATGACA-3' and Lwasa_odc1_R $5^{\prime}$-TTAATCAAGTCG AGATCATCTTCAGT-3' were designed, which have binding sites on contig00080 and contig00042, respectively. The PCR produced an amplicon of about $500 \mathrm{bp}$ as expected, confirming that the contigs are concatenated (data not shown). The complete gene was named $o d c 2$, and its sequence is deposited in the GenBank database under the accession number OL347710. Figure 2 represents how the two contigs are related, and the genes $o d c 1, o d c 2$, and potE.

\section{Database Searches and Similarity Trees}

The genetic organization odc2-odc1-potE (Figure 2) was found using BLAST searches against the genome sequence of other lactic acid bacteria, such as Levilactobacillus paucivorans DSM 22467 (GenBank ASM143712v1), Levilactobacillus lindianensis strain 220-4 (GenBank ASM394609v1), Levilactobacillus hunanensis strain 151-2B (GenBank ASM394614v1), and Lentilactobacillus kribbianus strain YH-lac9 (GenBank ASM1318407v1). To predict the preferred substrates of these putative decarboxylases, protein sequences with biochemically demonstrated functions, that is, ODC for ornithine decarboxylation, LDC for lysine decarboxylation, and AOLDC for the biosynthetic arginine/ornithine/lysine decarboxylation of Gram-positive bacteria, were selected (Table 2) and aligned with the Odc1 and Odc2 mentioned above. Similarities were visualized with a neighbor-joining tree (Figure 3A). The ODC and Odc2 amino acid sequences clustered together and were distinct from LDC and Odc1, which formed another cluster. The AOLDC protein sequences were apart from both the ODC and LDC clusters. Based on these results, we hypothesized that Odc1 from $P$. wasatchensis decarboxylates lysine to cadaverine, while Odc2 produces putrescine from ornithine. PotE is essential for the import of amino acid and the secretion of biogenic amines and is generally located next to the decarboxylase gene. The similarity tree for the reference PotE sequences (Figure 3B) showed two clusters, one with the PotE sequences associated with a unique ODC, as well as another one with PotE sequences associated with both ODC and LDC, such as, for example, $P$. wasatchensis.

\section{Decarboxylase Activity of Recombinant Proteins}

The two genes $o d c 1$ and $o d c 2$ were cloned into the expression vector pEG-His1 to study their function in more detail. The Sanger sequences of positive clones Odc1_Lw35 and Odc2_C19 confirmed the correct insertion of both the odc1 and odc2 genes, respectively. Cloning fused a His-tag to the C-terminus of the putative ornithine decarboxylases, which allowed the recombinant proteins to be purified by Ni-metal affinity chromatography. The E. coli expression strain BL21(DE3) also possesses ornithine and lysine decarboxylases. Therefore, the protein extract of E. coli BL21(DE3), which did not harbor a plasmid, was also separated by Ni-metal affinity chromatography. Neither ornithine decarboxylation nor lysine decarboxylation were detected in the fraction of this E. coli control extract that was eluted from the column material (data not shown). 
TABLE 2 | Accession number of reference sequences retrieved from GenBank for phylogenetic analysis.

\begin{tabular}{|c|c|c|c|c|c|}
\hline Organisms & Aoldc & Ldc/odc1 & Odc/odc2 & PotE & References \\
\hline Lacticaseibacillus paracasei & Q038E5 & & & & Romano et al., 2012 \\
\hline Lactobacillus gasseri & WP_011679015 & & & & Romano et al., 2012 \\
\hline Ligilactobacillus saerimneri & WP_040533848 & WP_009553967 & WP_009553942 & WP_009553966 & Romano et al., 2013b \\
\hline Oenococcus oeni & & & CAG34069 & CAM07323 & Romano et al., 2012 \\
\hline Furfurilactobacillus rossiae & & & ANJ65946 & ANJ65947 & Del Rio et al., 2018 \\
\hline Levilactobacillus brevis & & & AFC60624 & AFC60625 & Romano et al., 2012 \\
\hline Latilactobacillus curvatus & & & WP_039099122 & WP_039099123 & Li et al., 2018, 2019 \\
\hline Paucilactobacillus wasatchensis & & WP_044011228 & OL347710 & WP_044011229 & this study \\
\hline Levilactobacillus paucivorans & & WP_057879136 & WP_057879135 & WP_057879137 & unpublished data \\
\hline Staphylococcus epidermidis & & & ADJ57328 & ADJ57329 & Coton et al., 2010 \\
\hline Levilactobacillus lindianensis & & WP_125546544 & WP_125546542 & WP_125546546 & \\
\hline Lentilactobacillus kribbianus & & WP_172188509 & WP_172188507 & WP_172188511 & \\
\hline Levilactobacillus hunanensis & & WP_125574408 & WP_125574407 & WP_125574409 & \\
\hline
\end{tabular}

Only publications describing decarboxylase activity are mentioned under "References."

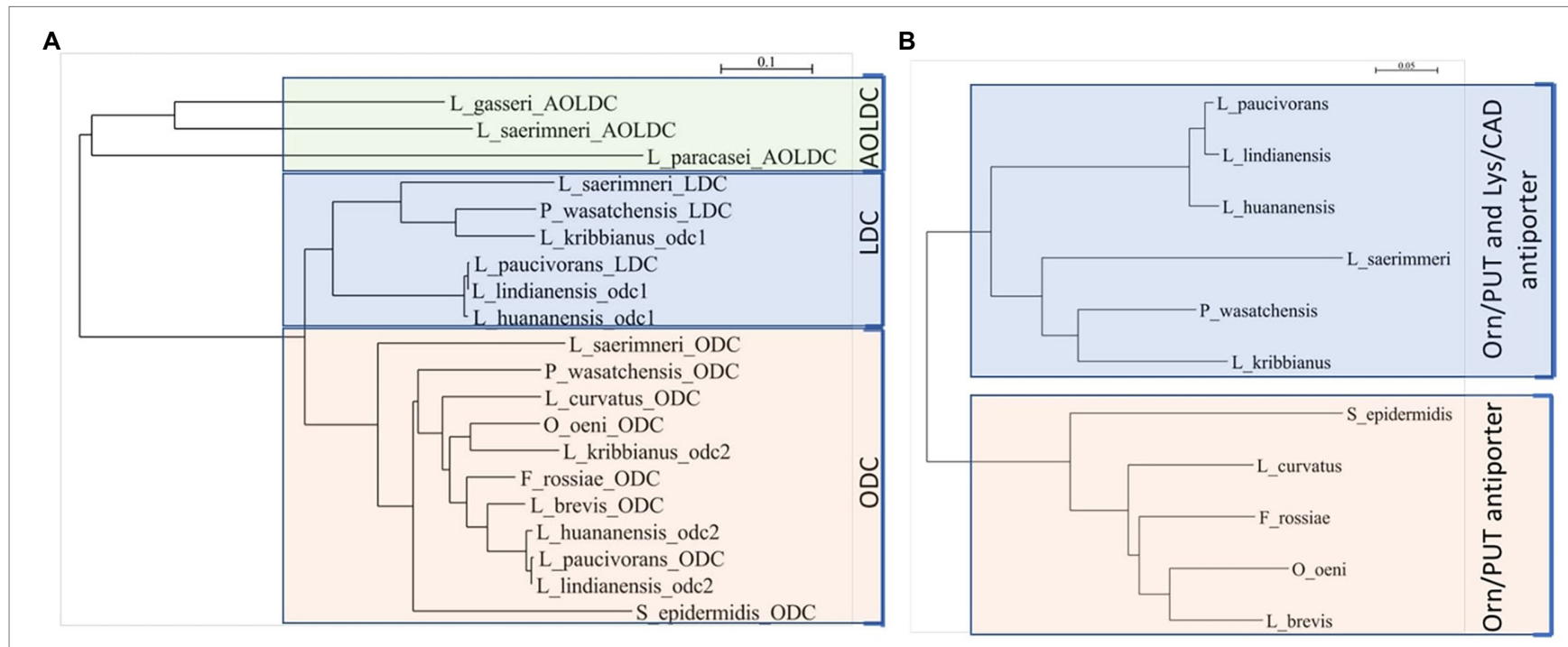

FIGURE 3 | Neighbor-joining trees based upon the alignment of amino acid sequences of representative decarboxylases (A) and associated antiporter (B) and the predicted function. ODC, ornithine decarboxylase; LDC, lysine decarboxylase; AOLDC, arginine/ornithine/lysine decarboxylase; PUT, putrescine; CAD, cadaverine; Orn, ornithine; and Lys, lysine.

For the enzyme tests used, it was found that Odc1_Lw35 decarboxylated lysine to cadaverine. No activity was detected for this enzyme with ornithine and arginine. Odc2_C19 decarboxylated both lysine and ornithine and the two products cadaverine and putrescine, respectively, were detected. Odc2_C19 showed no decarboxylating activity toward arginine.

When the $\mathrm{pH}$ dependence of the decarboxylation reaction was determined for both proteins, Odc1_Lw35 was found to be active in a narrower $\mathrm{pH}$ range than Odc2_C19 (Figure 4). Indeed, Odc1_Lw35 showed decarboxylation activity at $\mathrm{pH} 4.8$, 5.5, and 6.0 with an optimum at $\mathrm{pH}$ 5.5. Odc2_C19 showed detectable decarboxylation activity from $\mathrm{pH} 4.0$ to $\mathrm{pH} 7.4$ with the highest activity at $\mathrm{pH} 5.5$ and $\mathrm{pH}$ 6.0.

The kinetic parameters for both enzymes were determined at $\mathrm{pH}$ 5.5, a value that also occurs in cheese. The kinetic parameters for lysine as a substrate show that Odc1_Lw35 has a slightly lower $\mathrm{Km}$ value and a slightly higher kcat value than Odc2_C19 (Table 3). When the kinetic parameters of the two substrates ornithine and lysine were compared for Odc2_C19, the enzyme clearly preferred the former.

\section{DISCUSSION}

This study shows that the P. wasatchensis strain DSM 29958 produces cadaverine and putrescine in media supplemented with lysine and ornithine, respectively. Upon the addition of arginine to the culture medium, neither agmatine nor putrescine were observed, suggesting that there is no arginine decarboxylase activity or arginine deiminase (ADI) pathway to convert arginine into ornithine upstream of putrescine production, as described for the Weissella halotolerans strain W22 and L. rossiae strain 


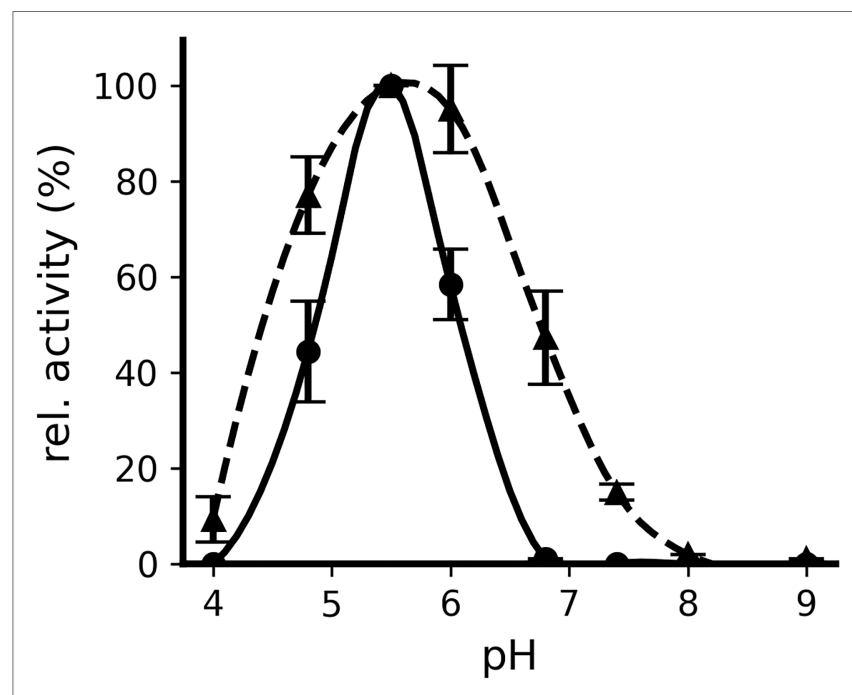

FIGURE 4 | Influence of pH on the decarboxylating activity of Odc1_Lw35 (-) and Odc2_C19 (A). The relative activity is illustrated with respect to the activity measured at $\mathrm{pH} 5.5$. Odc1_Lw35 was incubated with $10 \mathrm{mM}$ of L-lysine and Odc2_C19 with $10 \mathrm{mM}$ of L-ornithine. The illustration represents the mean ( \pm standard deviation) of triplicates.

TABLE 3 | Kinetic parameters of Odc1_Lw35 and Odc2_C19.

\begin{tabular}{llcc}
\hline Enzyme & Substrate & Km (mM) & kcat $\left(\mathbf{s}^{\mathbf{- 1}}\right)$ \\
\hline Odc1_Lw35\# & lysine & $16.0 \pm 1.8$ & $3.0 \pm 0.1$ \\
Odc2_C19 & lysine & $20.2 \pm-0.4$ & $1.8 \pm 0.1$ \\
Odc2_C19 & ornithine & $4.9 \pm 0.9$ & $275 \pm 43.37$ \\
\hline
\end{tabular}

Values represent the means of triplicates (Km. Michaelis-Menten constant; kcat: turnover number). "Odc1: decarboxylating activity toward ornithine was not detected.

D87 (Pereira et al., 2009; Del Rio et al., 2018). This is in line with the genomic data for DSM 29958, in which no gene is annotated as arginine deiminase.

There are a few points to note regarding agmatine. Figure 1 shows that the dansylated agmatine did not migrate in HPTLC using a solvent system made up with chloroform-triethylamine. This is consistent with the studies of Li et al. (2016) who used different solvent systems consisting of chloroformtriethylamine with and without diethyl ether. For all studied cases, the authors report a retention factor (Rf) value of 0 for agmatine. Romano et al. (2012) describe that they separated agmatine with a solvent system consisting of chloroformtriethylamine-methanol. Unfortunately, this study does not report an $\mathrm{Rf}$ value. The solvent system was also used in this study and, indeed, it was observed that the dansylated reference substance agmatine migrated a few millimeters (data not shown). However, it was not possible to clearly separate agmatine from the unknown compounds that also appeared in the culture supernatants (lane 2 in Figure 1). Therefore, the signal intensities of the dansylated compounds that were found in the fermented medium without and with arginine and that had the same Rf values as the dansylated agmatine were compared (for example: lane 2 and lane 5 in Figure 1). Since no clear different signal intensities were found, it was concluded that no agmatine was formed. This conclusion is supported by the absence of a gene encoding an arginine decarboxylase in the genome of $P$. wasatchensis.

Similarly, in trial cheese produced with an adjunct $P$. wasatchensis culture, $P$. wasatchensis could grow to approximately 7 log genome equivalents per gram and produce cadaverine and putrescine, while the amounts of the respective amino acid precursors were reduced in comparison with the control cheese. More cadaverine $\left(1,085 \mathrm{mg} \mathrm{kg}^{-1}\right)$ than putrescine (304 $\mathrm{mg} \mathrm{kg}^{-1}$ ) was measured, probably because the cheese contained more lysine than ornithine. Because casein does not contain ornithine, this amino acid is supposed to have been produced by Lactococcus lactis, which was present in the applied starter culture, via the ADI pathway (Fernández and Zúñiga, 2006; Linares et al., 2011). However, the relative amounts of biogenic amines produced could be influenced by other factors, such as $\mathrm{pH}$, redox potential, and the presence of cofactors (De Filippis et al., 2013; Durak-Dados et al., 2020).

P. wasatchensis is a spoilage organism associated with late gas production in Cheddar cheese. It produces gas in medium when galactose is present (Ortakci et al., 2015a). From this, it was deduced that the degradation of galactose in cheese can lead to increased gas formation. Indeed, cheese experiments showed that the addition of ribose and galactose in the presence of $P$. wasatchensis caused increased gas formation (Ortakci et al., 2015b). However, in the same study, gas formation was also observed in the cheeses with $P$. wasatchensis without the addition of sugar. Therefore, it can be concluded that other substrates are also responsible for the undesirable gas formation. In the cheese experiment of this study, cadaverine and putrescine were produced. The production of both substances resulted from the decarboxylation of lysine and ornithine, respectively. In can be calculated that the amount of cadaverine $\left(1,085 \mathrm{mg} \mathrm{kg}^{-1}\right)$ and putrescine $\left(304 \mathrm{mg} \mathrm{kg}^{-1}\right)$ could yield about $14 \mathrm{mmol} \mathrm{kg}^{-1}$ of carbon dioxide, which corresponds to approximately $0.31 \mathrm{~L} \mathrm{~kg}^{-1}$ of cheese assuming that the molar volume is $22.4 \mathrm{~L}$ at $0^{\circ} \mathrm{C}$ and $1 \mathrm{~atm}$.

Ortakci et al. (2015b) produced cheese in which galactose was added to the curd before pressing. In this way, the cheeses with the highest galactose content contained $0.238 \mathrm{~g} \mathrm{~kg}^{-1}$ at the beginning of ripening. Using the above mathematical calculation, the metabolization of galactose would produce $0.29 \mathrm{~L} \mathrm{~kg}^{-1}$. In summary, this means that both the degradation of galactose and amino acids can produce gas of the same magnitude. Since the glucose and the galactose resulting from lactose are consumed by the starter culture within the first $24 \mathrm{~h}$ in many cheese varieties, it is recommended to also determine the biogenic amines in Cheddar cheese affected by late blowing in future studies. This would give an indication of the extent to which amino acid decarboxylation is involved in the cheese defect.

To better understand the molecular basis of biogenic amine formation, the genomic data of $P$. wasatchensis were analyzed for the presence of genes encoding amino acid decarboxylases. We identified a gene cluster with three contiguous genes, odc2odc1-potE, which had similarities with the respective genes involved in the three-component lysine/ornithine decarboxylation 
system in Ligilactobacillus saerimneri 30a. The components are an ornithine decarboxylase, a lysine decarboxylase, and a transporter able to exchange lysine for cadaverine as well as ornithine for putrescine (Romano et al., 2012). Concerning $P$. wasatchensis, the heterologously expressed protein Odc1_Lw35 decarboxylated lysine to cadaverine. The recombinant protein Odc2_C19 preferentially produced putrescine from ornithine but could also produce cadaverine from lysine with a lower affinity. This lower affinity for lysine was also observed for the Odc enzyme of L. saerimneri 30a by Romano et al. (2013b). The catalytic efficiency of Odc2_C19 was about 600-fold higher with ornithine as compared to lysine, but secondary lysine decarboxylase activity was clearly demonstrated. In the trial cheese, cadaverine was measured after 120 days of ripening, but the contributions of both enzymes cannot be assessed with wild-type bacteria. These contributions could further be investigated if knock-out strains were available.

However, given the contiguous presence of two similar genes with similar functions, one of them conferring an additional side-function identical to the function of the second gene could be a perfect example of the InnovationAmplification-Divergence (IAD) model (Bergthorsson et al., 2007). This model explains the origin of new genes via selection pressure. Concretely, it suggests that, under selection for lysine decarboxylation, gene coding for ornithine decarboxylase with side lysine decarboxylation activity (odc2) is duplicated to increase the overall lysine decarboxylation activity. This new copy ( odc1) evolves to improve LDC activity. This process may be particularly advantageous for adaptation to a new ecological niche, for example, when moving from an ornithine-rich environment to a lysine-rich one. The ability to decarboxylate lysine to cadaverine could allow for better resistance to an acidic $\mathrm{pH}$, as demonstrated for ornithine decarboxylation by $L$. rossiae (Del Rio et al., 2018), or to osmotic stress through tyrosine decarboxylation by Streptococcus thermophilus (La Gioia et al., 2011). Finally, the decarboxylation of amino acids as an alternative energy source is often mentioned but has not been universally demonstrated (Cid et al., 2008; Del Rio et al., 2018). Possessing genes coding

\section{REFERENCES}

Altschul, S. F., Madden, T. L., Schäffer, A. A., Zhang, J., Zhang, Z., Miller, W., et al. (1997). Gapped BLAST and PSI-BLAST: a new generation of protein database search programs. Nucleic Acids Res. 25, 3389-3402. doi: 10.1093/nar/25.17.3389

Ascone, P., Maurer, J., Haldemann, J., Irmler, S., Berthoud, H., Portmann, R., et al. (2017). Prevalence and diversity of histamine-forming Lactobacillus parabuchneri strains in raw milk and cheese - a case study. Int. Dairy J. 70, 26-33. doi: 10.1016/j.idairyj.2016.11.012

Bachmann, H. P., and Spahr, U. (1995). The fate of potentially pathogenic bacteria in Swiss hard and semihard cheeses made from raw milk. J. Dairy Sci. 78, 476-483. doi: 10.3168/jds.S0022-0302(95)76657-7

Benkerroum, N. (2016). Biogenic amines in dairy products: origin, incidence, and control means. Compr. Rev. Food Sci. Food Saf. 15, 801-826. doi: 10.1111/1541-4337.12212

Bergthorsson, U., Andersson, D. I., and Roth, J. R. (2007). Ohno's dilemma: evolution of new genes under continuous selection. Proc. Natl. Acad. Sci. U. S. A. 104, 17004-17009. doi: 10.1073/pnas.0707158104

Berthoud, H., Wüthrich, D., Bruggmann, R., Wechsler, D., Fröhlich-Wyder, M.T., and Irmler, S. (2017). Development of new methods for the quantitative for amino acid decarboxylation seems to be advantageous, but such genes are generally not universally distributed in a species. Guerrini et al. (2002) reported that $16 \%$ of Oenococcus oeni had the capability to form ornithine. Evidence of horizontal transfers was reported (Coton and Coton, 2009; La Gioia et al., 2011; Romano et al., 2014; Wüthrich et al., 2017; Del Rio et al., 2018). It would be interesting to evaluate the potential of other $P$. wasatchensis strains to form biogenic amines. In a general sense, screening bacteria for their potential diamines production could be made more successful by searching for the potE gene because this avoids the detection of constitutive decarboxylases (Aoldc). The substrate affinity of decarboxylases must be determined biochemically. However, with pheno- and genotypic data from more strains, substrate specificity prediction should become more precise.

In summary, this study showed the ability of $P$. wasatchensis to produce both cadaverine and putrescine in culture medium and in a trial cheese. Lysine decarboxylase genes of lactic acid bacteria could explain very high amounts of cadaverine $\left(>1,000 \mathrm{mg} \mathrm{kg}^{-1}\right)$ in cheese or other fermented products and should be further investigated.

\section{DATA AVAILABILITY STATEMENT}

The datasets presented in this study can be found in online repositories. The names of the repository/repositories and accession number(s) can be found at: https://www.ncbi.nlm. nih.gov/genbank/, OL347710.

\section{AUTHOR CONTRIBUTIONS}

HB, DW, and SI contributed to conception and design of the study. DW designed the cheese trial. SI performed the biochemical analysis and wrote sections of the manuscript. HB performed the molecular biology analysis and wrote the first draft of the manuscript. All authors contributed to the article and approved the submitted version.

detection and typing of Lactobacillus parabuchneri in dairy products. Int Dairy J. 70, 65-71. doi: 10.1016/j.idairyj.2016.10.005

Cid, S. B., Jesús Miguélez-Arrizado, M., Becker, B., Holzapfel, W. H., and Carmen Vidal-Carou, M. (2008). Amino acid decarboxylation by Lactobacillus curvatus CTC273 affected by the $\mathrm{pH}$ and glucose availability. Food Microbiol. 25, 269-277. doi: 10.1016/j.fm.2007.10.013

Coton, E., and Coton, M. (2009). Evidence of horizontal transfer as origin of strain to strain variation of the tyramine production trait in Lactobacillus brevis. Food Microbiol. 26, 52-57. doi: 10.1016/j.fm.2008.07.009

Coton, M., Delbés-Paus, C., Irlinger, F., Desmasures, N., Le Fleche, A., Stahl, V., et al. (2012). Diversity and assessment of potential risk factors of Gramnegative isolates associated with French cheeses. Food Microbiol. 29, 88-98. doi: 10.1016/j.fm.2011.08.020

Coton, E., Mulder, N., Coton, M., Pochet, S., Trip, H., and Lolkema, J. S. (2010). Origin of the putrescine-producing ability of the coagulase-negative bacterium Staphylococcus epidermidis 2015B. Appl. Environ. Microbiol. 76, 5570-5576. doi: 10.1128/AEM.00441-10

De Filippis, F., Pennacchia, C., Di Pasqua, R., Fiore, A., Fogliano, V., Villani, F., et al. (2013). Decarboxylase gene expression and cadaverine 
and putrescine production by Serratia proteamaculans in vitro and in beef. Int. J. Food Microbiol. 165, 332-338. doi: 10.1016/j. ijfoodmicro.2013.05.021

Delbès-Paus, C., Pochet, S., Helinck, S., Veisseire, P., Bord, C., Lebecque, A., et al. (2012). Impact of Gram-negative bacteria in interaction with a complex microbial consortium on biogenic amine content and sensory characteristics of an uncooked pressed cheese. Food Microbiol. 30, 74-82. doi: 10.1016/j. fm.2011.12.008

Del Rio, B., Alvarez-Sieiro, P., Redruello, B., Martin, M. C., Fernandez, M., Ladero, V., et al. (2018). Lactobacillus rossiae strain isolated from sourdough produces putrescine from arginine. Sci. Rep. 8:3989. doi: 10.1038/ s41598-018-22309-6

Dimidi, E., Cox, S. R., Rossi, M., and Whelan, K. (2019). Fermented foods: definitions and characteristics, impact on the gut microbiota and effects on gastrointestinal health and disease. Nutrients 11:1806. doi: 10.3390/nu11081806

Dreier, M., Berthoud, H., Shani, N., Wechsler, D., and Junier, P. (2020). Development of a high-throughput microfluidic qPCR system for the quantitative determination of quality-relevant bacteria in cheese. Front. Microbiol. 11:619166. doi: 10.3389/fmicb.2020.619166

Durak-Dados, A., Michalski, M., and Osek, J. (2020). Histamine and other biogenic amines in food. J. Vet. Res. 64, 281-288. doi: 10.2478/jvetres-2020-0029

Egger, L., Ménard, O., Abbühl, L., Duerr, D., Stoffers, H., Berthoud, H., et al. (2021). Higher microbial diversity in raw than in pasteurized milk Raclettetype cheese enhances peptide and metabolite diversity after in vitro digestion. Food Chem. 340:128154. doi: 10.1016/j.foodchem.2020.128154

Fernández, M., and Zúñiga, M. (2006). Amino acid catabolic pathways of lactic acid bacteria. Crit. Rev. Microbiol. 32, 155-183. doi: 10.1080/10408410600880643

Gille, D., Schmid, A., Walther, B., and Vergères, G. (2018). Fermented food and non-communicable chronic diseases: a review. Nutrients 10:448. doi: $10.3390 /$ nu10040448

Gouy, M., Guindon, S., and Gascuel, O. (2010). SeaView version 4: a multiplatform graphical user interface for sequence alignment and phylogenetic tree building. Mol. Biol. Evol. 27, 221-224. doi: 10.1093/molbev/msp259

Guerrini, S., Mangani, S., Granchi, L., and Vincenzini, M. (2002). Biogenic amine production by Oenococcus oeni. Curr. Microbiol. 44, 374-378. doi: 10.1007/s00284-001-0021-9

La Gioia, F., Rizzotti, L., Rossi, F., Gardini, F., Tabanelli, G., and Torriani, S. (2011). Identification of a tyrosine decarboxylase gene (tdcA) in Streptococcus thermophilus 1TT45 and analysis of its expression and tyramine production in milk. Appl. Environ. Microbiol. 77, 1140-1144. doi: 10.1128/ AEM.01928-10

Li, S., Johansson, M., Vidanarachchi, J. K., Pickova, J., and Zamaratskaia, G. (2016). Determination of biogenic amines in aerobically stored beef using high-performance thin-layer chromatography densitometry. Acta Agric. Scand. A Anim. Sci. 66, 199-205. doi: 10.1080/09064702.2017.1315455

Li, L., Wen, X., Wen, Z., Chen, S., Wang, L., and Wei, X. (2018). Evaluation of the biogenic amines formation and degradation abilities of Lactobacillus curvatus from Chinese bacon. Front. Microbiol. 9:1015. doi: 10.3389/ fmicb.2018.01015

Li, L., Zou, D., Ruan, L., Wen, Z., Chen, S., Xu, L., et al. (2019). Evaluation of the biogenic amines and microbial contribution in traditional Chinese sausages. Front. Microbiol. 10:872. doi: 10.3389/fmicb.2019.00872

Linares, D. M., Martín, M. C., Ladero, V., Alvarez, M. A., and Fernández, M. (2011). Biogenic amines in dairy products. Crit. Rev. Food Sci. Nutr. 51, 691-703. doi: 10.1080/10408398.2011.582813

Maher, M. M., Jordan, K. N., Upton, M. E., and Coffey, A. (2001). Growth and survival of E. coli O157:H7 during the manufacture and ripening of a smear-ripened cheese produced from raw milk. J. Appl. Microbiol. 90, 201-207. doi: 10.1046/j.1365-2672.2001.01232.x

Man, J. C. D., De Man, J. C., Rogosa, M., and Elisabeth Sharpe, M. (1960). A medium for the cultivation of Lactobacilli. J. Appl. Bacteriol. 23, 130-135. doi: 10.1111/j.1365-2672.1960.tb00188.x

McMahon, D. J., Bowen, I. B., Green, I., Domek, M., and Oberg, C. J. (2020). Growth and survival characteristics of Paucilactobacillus wasatchensis WDCO4. J. Dairy Sci. 103, 8771-8781. doi: 10.3168/jds.2020-18597

Montel, M.-C., Buchin, S., Mallet, A., Delbes-Paus, C., Vuitton, D. A., Desmasures, N., et al. (2014). Traditional cheeses: rich and diverse microbiota with associated benefits. Int. J. Food Microbiol. 177, 136-154. doi: 10.1016/j. ijfoodmicro.2014.02.019
Oberg, C. J., Culumber, M. D., Allen, T., Oberg, T. S., Villalba, B., and McMahon, D. J. (2016). 0546 characterization of Lactobacillus wasatchensis from aged cheeses showing late-gas defects. J. Anim. Sci. 94, 261-261. doi: 10.2527/jam2016-0546

Ortakci, F., Broadbent, J. R., Oberg, C. J., and McMahon, D. J. (2015a). Growth and gas production of a novel obligatory heterofermentative Cheddar cheese nonstarter lactobacilli species on ribose and galactose. J. Dairy Sci. 98, 3645-3654. doi: 10.3168/jds.2014-9293

Ortakci, F., Broadbent, J. R., Oberg, C. J., and McMahon, D. J. (2015b). Late blowing of Cheddar cheese induced by accelerated ripening and ribose and galactose supplementation in presence of a novel obligatory heterofermentative nonstarter Lactobacillus wasatchensis. J. Dairy Sci. 98, 7460-7472. doi: 10.3168/ jds.2015-9468

O'Sullivan, D. J., Fallico, V., O'Sullivan, O., McSweeney, P. L. H., Sheehan, J. J., Cotter, P. D., et al. (2015). High-throughput DNA sequencing to survey bacterial histidine and tyrosine decarboxylases in raw milk cheeses. BMC Microbiol. 15:266. doi: 10.1186/s12866-015-0596-0

Pereira, C. I., San Romão, M. V., Lolkema, J. S., and Crespo, M. T. B. (2009). Weissella halotolerans W22 combines arginine deiminase and ornithine decarboxylation pathways and converts arginine to putrescine. J. Appl. Microbiol. 107, 1894-1902. doi: 10.1111/j.1365-2672.2009.04371.x

Pessione, E., Mazzoli, R., Giuffrida, M. G., Lamberti, C., Garcia-Moruno, E., Barello, C., et al. (2005). A proteomic approach to studying biogenic amine producing lactic acid bacteria. Proteomics 5, 687-698. doi: 10.1002/ pmic.200401116

Rios, E. A., Ramos-Pereira, J., Santos, J. A., López-Díaz, T. M., Otero, A., and Rodríguez-Calleja, J. M. (2020). Behaviour of non-O157 STEC and atypical EPEC during the manufacturing and ripening of raw milk cheese. Foods 9:1215. doi: 10.3390/foods9091215

Romano, A., Ladero, V., Alvarez, M. A., and Lucas, P. M. (2014). Putrescine production via the ornithine decarboxylation pathway improves the acid stress survival of Lactobacillus brevis and is part of a horizontally transferred acid resistance locus. Int. J. Food Microbiol. 175, 14-19. doi: 10.1016/j. ijfoodmicro.2014.01.009

Romano, A., Trip, H., Campbell-Sills, H., Bouchez, O., Sherman, D., Lolkema, J. S., et al. (2013a). Genome sequence of Lactobacillus saerimneri 30a (formerly Lactobacillus sp. strain 30a), a reference lactic acid bacterium strain producing biogenic amines. Genome Announc. 1:e00097-12. doi: 10.1128/ genomeA.00097-12

Romano, A., Trip, H., Lolkema, J. S., and Lucas, P. M. (2013b). Three-component lysine/ornithine decarboxylation system in Lactobacillus saerimneri 30a. $J$. Bacteriol. 195, 1249-1254. doi: 10.1128/JB.02070-12

Romano, A., Trip, H., Lonvaud-Funel, A., Lolkema, J. S., and Lucas, P. M. (2012). Evidence of two functionally distinct ornithine decarboxylation systems in lactic acid bacteria. Appl. Environ. Microbiol. 78, 1953-1961. doi: 10.1128/AEM.07161-11

Schirone, M., Tofalo, R., Fasoli, G., Perpetuini, G., Corsetti, A., Manetta, A. C., et al. (2013). High content of biogenic amines in pecorino cheeses. Food Microbiol. 34, 137-144. doi: 10.1016/j.fm.2012.11.022

Schirone, M., Tofalo, R., Visciano, P., Corsetti, A., and Suzzi, G. (2012). Biogenic amines in Italian pecorino cheese. Front. Microbiol. 3:171. doi: 10.3389/ fmicb.2012.00171

Sievers, F., Wilm, A., Dineen, D., Gibson, T. J., Karplus, K., Li, W., et al. (2011). Fast, scalable generation of high-quality protein multiple sequence alignments using clustal omega. Mol. Syst. Biol. 7:539. doi: 10.1038/msb.2011.75

Tofalo, R., Perpetuini, G., Battistelli, N., Pepe, A., Ianni, A., Martino, G., et al. (2019). Accumulation aminobutyric acid and biogenic amines in a traditional raw milk ewe's cheese. Foods 8:401. doi: 10.3390/foods8090401

Virtanen, P., Gommers, R., Oliphant, T. E., Haberland, M., Reddy, T., Cournapeau, D., et al. (2020). SciPy 1.0: fundamental algorithms for scientific computing in Python. Nat. Methods 17, 261-272. doi: 10.1038/s41592-019-0686-2

Wenzel, C., Irmler, S., Bisig, W., Guggisberg, D., Roetschi, A., Portmann, R., et al. (2018). The effect of starters with a functional arginine deiminase pathway on cheese ripening and quality. Int. Dairy J. 85, 191-200. doi: 10.1016/j.idairyj.2018.05.008

Wüthrich, D., Berthoud, H., Wechsler, D., Eugster, E., Irmler, S., and Bruggmann, R. (2017). The histidine decarboxylase gene cluster of Lactobacillus parabuchneri was gained by horizontal gene transfer and is mobile within the species. Front. Microbiol. 8:218. doi: 10.3389/ fmicb. 2017.00218 
Zhang, K., and Ni, Y. (2014). Tyrosine decarboxylase from Lactobacillus brevis: soluble expression and characterization. Protein Expr. Purif. 94, 33-39. doi: 10.1016/j.pep. 2013.10 .018

Conflict of Interest: HB, DW, and SI were employed by Agroscope.

Publisher's Note: All claims expressed in this article are solely those of the authors and do not necessarily represent those of their affiliated organizations, or those of the publisher, the editors and the reviewers. Any product that may be evaluated in this article, or claim that may be made by its manufacturer, is not guaranteed or endorsed by the publisher.

Copyright (c) 2022 Berthoud, Wechsler and Irmler. This is an open-access article distributed under the terms of the Creative Commons Attribution License (CC BY). The use, distribution or reproduction in other forums is permitted, provided the original author(s) and the copyright owner(s) are credited and that the original publication in this journal is cited, in accordance with accepted academic practice. No use, distribution or reproduction is permitted which does not comply with these terms. 